\title{
Quantification of peripapillary RNFL thickness with the help of OCT in normal, ocular hypertensive and glaucomatous eyes
}

\author{
Mairembam Ranjana ${ }^{1}$, Kamson Anthony ${ }^{2, *}$, Rajkumar Vidyarani ${ }^{3}$ \\ ${ }^{\mathbf{1}}$ Senior Resident, ${ }^{\mathbf{2}}$ Assistant Professor, ${ }^{\mathbf{3}}$ Associate Professor, Dept. of Ophthalmology, Jawaharlal Nehru Institute of Medical \\ Sciences (JNIMS), Guwahati, India
}

*Corresponding Author:

Email: anthonyjnims@gmail.com

\begin{abstract}
s
Aims: To quantify and compare the retinal nerve fiber thickness in normal, ocular hypertensive and glaucomatous eyes using Optical coherence tomography (OCT).

Materials and Methods: Patients were divided in to three groups of 30 each for normal, ocular hypertensive and primary open angle glaucoma. Inclusion criteria were of patients between 30-70 years of age, visual acuity of 6/36 or better, Refractive error not exceeding 5D and no prior surgery. Complete eye evaluation and was done followed by OCT Scan using the protocol "Fast RNFL thickness". RNFL thickness was assessed in 4 quadrants and statistical analysis was done using student t test and analysis of variance (ANOVA).

Results: RNFL was significantly thinner in OHT eyes than in normal eyes specifically in the inferior and nasal quadrants. $12.6 \%$ decrease in RNFL thickness was seen in OHT eyes as compared to normal eyes and about $22.29 \%$ decrease in RNFL thickness in POAG eyes as compared to OHT.

Conclusions: Incorporation of OCT in glaucoma practice can helped us in evaluation of optic disc, quantifying of RNFL, identifying the structural changes in RNFL and the progression of disease. The magnitude of focal RNFL thinning in OCT was related to the magnitude of decreased global indices sensitivity in glaucomatous patient.
\end{abstract}

Keywords: Low coherence optical tomography, Optic disc, Retinal nerve fiber layer, Visual field.

\section{Introduction}

World Health Organization (WHO) in 2000 indicate that glaucoma is the $2^{\text {nd }}$ largest cause of irreversible blindness with 6.4 million persons, behind cataract's 19 million. ${ }^{1}$ Recent study shows that 8.4 million people who are blind as the result of glaucoma. These numbers are set to increase to 80 million and 11.2 million by $2020 .^{28}$

Diagnosing a case of glaucoma in the clinics are limited by factors like observer dependent, variability, subjectivity, age, gender, race and time of examination. Glaucomatous Visual field defects are detected when more than $60 \%$ damage to RNFL has already occurred. So many a times glaucoma goes undiagnosed or misinterpretation finally costing the patients his vision for ever. Studies have shown that a decrease in RNFL thickness usually precedes visual field loss and optic nerve head defects. ${ }^{2-5}$

Newer diagnostic tools like OCT (low coherence optical tomography) have helped us in detecting as well as study the progression and structural changes associated with glaucomatous. It is a non-invasive, noncontact transpupillary imaging technique, working on the principle of low coherence interferometry that generates retinal tomography with less than $10 \mu$ axial and $20 \mu$ transverse resolutions.

The optical characteristic of the RNFL which corresponds to its internal reflectivity provides additional information regarding diffuse and localized thinning thus helping us in quantify RNFL loss in patients who are at risk of developing glaucoma.

\section{Materials and Methods}

This study was conducted for a period of 2 years between 2014 to 2015 from the Northeastern population of India who came to attend our eye OPD. After Informed consent the participants is divided into three groups consisting of 30 patients each for normal, ocular hypertensive and POAG.

Group 1 consists of 30 normal patients with open angle, IOP of less than $21 \mathrm{mmHg}$ in 2 measurements, $\mathrm{CD}$ ratio less than 0.2 , normal visual field. Group 2 consist of 30 patients of OHT with IOP of more than $21 \mathrm{mmHg}$ in of 2 different measurements, normal of optic disc and visual field defects. Group 3 consist of 30 POAG patients with open angle, IOP of more than $21 \mathrm{mmHg}$ on two occasions, $\mathrm{CD}$ ratio of more than 0.6 with glaucomatous optic disc and visual field changes.

After complete ophthalmological examination Optical coherence tomography scan was done to measure the retinal nerve fiber layer (RNFL).Three circular scans, centered on the optic nerve head, each with $3.4 \mathrm{~mm}$ diameter, were acquired following pupillary dilatation. RNFL thickness was assessed in all the 4 retinal quadrants using the analysis protocol "Fast RNFL thickness" and the data analyzed with version 4.0software. Statistical analysis was done using the SPSS/PC (Sigma plot 2000).

The mean RNFL thickness among the groups was compared using student $t$ test and analysis of variance (ANOVA). The Pearson correlation co-efficient was used to establish the significance of association between global indices of field examination data (MD and PSD) and the RNFL thickness. 


\section{Results}

Mean RNFL was significantly thinner in ocular hypertensive eyes, $(72.8 \mu \mathrm{m})$ than in normal eyes, $(85.8$ $\mu \mathrm{m})$. There was significantly thinner of RNFL in ocular hypertensive eyes than in normal eyes in the inferior quadrant, $84.8 \mu \mathrm{m}(75.6-94.0 \mu \mathrm{m})$ vs $107.6 \mu \mathrm{m}(99.3-$ $115.9 \mu \mathrm{m})$; and in the nasal quadrant, $44.1 \mu \mathrm{m}(37.5-$ $51.7 \mu \mathrm{m})$ vs $61.8 \mu \mathrm{m}(53.0-65.6 \mu \mathrm{m})$. Thinning of RNFL is also seen more in glaucomatous eyes than in ocular hypertensive and normal eyes in all the quadrants. The descriptive characteristic of the subjects are summarized in Table 1.

Data from the three study groups (normal, OHT and glaucomatous) do not differed significantly according to the age $(\mathrm{p}=0.4237)$.The ocular hypertensive group on perimetry, shows that the mean RNFL thickness has a statistical correlation $(\mathrm{p}=0.002)$ with MD only and not with PSD. (Table 2)

RNFL thickness in the normal group followed the ISNT rule. There was considerable within-group variability in measured RNFL thickness. Average RNFL was significantly thinner in OHT eyes than in normal eyes. Specifically, RNFL in inferior and nasal quadrants are thinner in OHT eyes than in normal eyes. Though RNFL reduction was noted in all areas in glaucoma group as compared to OHT group, significant reduction was seen in the superior and inferior quadrants. (Table 3-5)

In this study, we have seen an average decrease in RNFL thickness of about $12.6 \%$ in OHT eyes as compared to normal eyes and about $22.29 \%$ decrease in POAG eyes as compared to OHT.

Our study, along with other studies on Asian eyes, has demonstrated that normal RNFL measurements in Asians are different from that reported from normal white eyes. Interpretation and risk of developing glaucoma must cautiously be based upon the normative database representative of the Population. (Table 5)

\section{Discussion}

The OCT is capable of producing high resolution cross-sectional images of the different retinal layers. The potential usefulness of the OCT in diagnosing and managing glaucoma is being evaluated and several cross sectional studies have already reported rationals for using OCT in glaucoma clinics. ${ }^{12,26}$

Demographic indicators, influence of age and sex: In our study group, most of them were from 30-70 years with the mean age \pm SD of $55 \pm 9.95$ years in POAG group, $51.6 \pm 9.77$ years in OHT and $52.8 \pm 10.6$ years in normal group.

Most glaucoma patients were older in age than patients with normal eyes and OHT, but the differences was not significant, avoiding the correction for age ( $\mathrm{P}=0.4237$ with d.f 2,87 by ANOVA.

Relation between Intraocular pressure and central corneal thickness: In our study the overall mean $\mathrm{IOP} \pm \mathrm{SD}$ (in $\mathrm{mmHg}$ ) in OHT group was $24.93 \pm 2.709$ while in POAG group it was $25.90 \pm 4.212(\mathrm{P}=0.16)$. In a study conducted by RP $\mathrm{Copt}^{2}$ mean $\mathrm{IOP} \pm \mathrm{SD}$ was higher in of OHT group $(27.13 \pm 3.84)$ compared to POAG group (23.33 \pm 7.10 ). The difference may be due to their prior used of topical anti-glaucoma medications while our group was not on any medication.

The mean $\mathrm{CCT} \pm \mathrm{SD}$ of OHT group was $555.266 \pm 32.109$ while POAG was $517.866 \pm 37.780 \mu \mathrm{m}$ but on comparison minimal significance was seen $(\mathrm{p}=0.029)$. while study by RP $\mathrm{Copt}^{2}$ at al found a significant $\mathrm{p}$ value for the mean $\mathrm{CCT} \pm \mathrm{SD}$ between the two groups $(\mathrm{p}<0.001)$.

The mean $\mathrm{CD}$ ratio $\pm \mathrm{SD}$ of normal, OHT and POAG were $0.268 \pm 0.084, \quad 0.383 \pm 0.075$ and $0.788 \pm 0.113$ respectively and on comparison it was found to have high statistical significance $(\mathrm{p}=<0.001)$. In a study conducted by Felipe ${ }^{3}$ et al, a similar value of baseline $\mathrm{VCD} \pm \mathrm{SD}$ of $0.7 \pm 0.09$ was found among the borderline ocular hypertensive and those that developing visual field loss subsequently were converters. This similarity in $\mathrm{VCD} \pm \mathrm{SD}$ points to the development of visual defects particularly in cases that had thinner corneas in comparison to normal CCT.

Relation between visual function and retinal nerve fiber layer thickness: $\mathrm{Hoh}^{4}$ et al showed a significant relationship between RNFL thickness and SAP (standard automated perimetry) MD (mean deviation) and corrected PSD measures in glaucomatous eyes $(r=0.68, r=0.59)$. In a similar study conducted by the El Beltagi ${ }^{5}$ et al, they found that the strength of Association between mean global RNFL thickness and SAP PSD was $\mathrm{R}^{2}=0.20$.

In a study done by Vandana Badlani ${ }^{6}$ et al they concluded that a linear correlation was found between RNFL and MD in early $(\mathrm{r}=0.6, \quad \mathrm{p}<0.001)$ and advanced( $r=0.5, p=0.007)$ glaucomatous hemifield.

Soliman and associates ${ }^{7}$ reported a significant correlation coefficient $(\mathrm{r}=0.557)$ between average RNFL thickness and mean deviation (MD) on W/W perimetry.

Zangwill $^{8}$ et al had also reported a significant correlation between average retinal nerve fiber layer (RNFL) thickness and mean deviation (MD).

Kanamori $^{10}$ et al showed that the highest correlation coefficient in all parameters was 0.729 at the average RNFL thickness suggesting that average RNFL thickness was most useful for monitoring glaucoma. In our study it was 0.72 .

But In our study the strength of the association between mean global RNFL thickness and SAP PSD was negatively related, $r=-0.53$ whereas with SAP MD it was found to be positively related, $\mathrm{r}=0.72$.

Significant decrease in average RNFL thickness of about $12.61 \%$ was seen in OHT eyes as compared to normal eyes and about $22.29 \%$ decrease in POAG eyes as compared to OHT.

Using stereo photogrammetric measures, Schwartz and Takamoto ${ }^{11}$ showed a significant decrease in RNFL 
thickness of about $18 \%$ in OHT eyes when compared with normal eyes. Comparison of RNFL thickness measurement in OHT and POAG patients from different studies is shown in table 6,7.

Results in some studies were contradictory for example Iester $^{12}$ et al failed to find significant difference between OHT and normal eyes. The difference seen in the above may be related to sensitivity of the measurement instrument, sample size, or difference in study population and differences in the criteria used to select the study population.

For instance, Iester ${ }^{12}$ et al used confocal scanning laser ophthalmoscope (HRT) to measure retinal height among other parameters and they found no significant between OHT and normal eye. These findings might be due to difference in sensitivity between OCT and HRT to measure RNFL thickness.

Negative results seen in Lachkar and Cohn ${ }^{12}$ might be due to the relatively small sample size used in the study. Our study found a significant thinning of RNFL in glaucomatous eyes as compared to OHT and normal eyes. The reason may be because we have included glaucomatous appearance of the optic disc and RNFL thickness as a criterion for glaucoma diagnosis in our study.

Most of the studies using the Stratus OCT have identified the average and inferior average RNFL thickness as the best discriminators between normal and glaucomatous eyes. ${ }^{13-21}$ Leung $^{22}$ et al demonstrated that the inferior peripapillary RNFL thickness had the best performance in discriminating glaucoma and glaucoma suspects. Bowde $\mathrm{C}^{19}$ et al showed significant differences in the average inferior RNFL thickness between normal and OHTs. Compared to normal eye significant thinning of RNFL was also seen in glaucomatous and OHT in all the four quadrants. In our study the global average, inferior average and nasal average RNFL measurements were significantly thinner in OHTs as compared to the normal controls.

But our study showed a significant difference in the RNFL thickness between OHT and normal eyes which may be the evidence of early damage to optic nerve. There is a possible that patients with OHT eyes with RNFL thickness measurements similar to those with glaucoma are greater risk of developing glaucomatous visual field defects. Similarly it is possible that OHT eyes in our study and those in other studies are early glaucomatous eyes.

\section{Conclusion}

The magnitude of focal RNFL thinning was related to the magnitude of decreased global indices sensitivity in glaucomatous patient in our study population. These findings suggest that measurement of RNFL thickness can help us in detection of glaucomatous damage even in a person with negligible visual field defect. Hence OCT scanning of RNFL is emerging as an important tool in the early diagnosis and long term management of glaucoma where other basic diagnostic test has failed of detecting glaucoma cases in its initial stage. Our study does have some limitation as the data are only of a particular population and it may not reflect the RNFL characters of the populations as a whole. Even early glaucomatous might have been missed out by standard automated perimetry which is not very sensitive to early functional damage. However we believed that these limitations do not impede reaching our results, similar to those from previous studies and conclusions. Further longitudinal studies should be conducted to determine if these differences in the results could increase the likelihood of conversion from normal to glaucomatous visual fields for patients with ocular hypertension (OHT).

\section{References}

1. WHO (2000). Strategic plan for vision 2020: The Right to sight, elimination of avoidable blindness in the SouthEast Asia region, New Delhi.

2. Copt R P, Thomas R, Mermoud A (1999): corneal thickness in ocular hypertension, POAG and NTG. Arch Ophthalmol 117(1):14-16.

3. Medeiros FA, Sample PA, Weinreb RN (2003): Corneal thickness measurements and visual function abnormalities in Ocular Hypertensive patients. Am J Ophthalmol 135 (2):131-137.

4. Hoh ST et al. (2000): OCT and scanning laser polarimetry in normal, ocular- hypertensive, and glaucomatous eyes. Am J Ophthalmol 129:129-35.

5. El Beltagi et al. (2003): RNFL thickness measured with OCT is related to visual function in glaucomatous eyes. 110(11):2185-91.

6. Badlani et al. (2006): Nerve fiber layer thickness in Glaucoma Patients with Assymetric Hemifield visual field loss. J Glaucoma. 15(4) 275-280.

7. Soliman MA et al. (2002): Retinal nerve fiber layer Analysis: Relationship between optical coherence tomography and red free photography. Am J Ophthalmol. 133(2):187-95.

8. Bowde C et al. (2001): Detecting early glaucoma by assessment of retinal nerve fiber layer thickness and visual function. Invest Ophthalmos Vis Sci 42(9):19932003.

9. Kanamori A et al. (2003): evaluation of the glaucomatous damage on retinal nerve fiber layer thickness measured by optical coherence tomography. Am j ophthalmol. 135(4):513-520.

10. P Brusini et al. (2008): Stratus-OCT imaging in early glaucomatous and in ocular hypertensive patients with and without frequency-doubling technology abnormalities. Eye.22:406-413.

11. Schwarttzs B Takamoto T (1992): Measurement of RNFL thickness and its functional correlation with the visual field. Bull Soc Belge Ophthalmol. 244:61-72.

12. Lachkar Y, Cohn H (1997): Nerve fiber layer analysis and optic disc parameter with the glaucoma-scope. Int Ophthalmol 1996-1997;20(1-3):33-38.

13. Medeiros F.A et al. (2004): Comparision of the GDxVCC scanning laser polarmeter, HRT II confocal scanning laser ophthalmoscope, and stratus OCT optical coherence tomography for the detection of glaucoma. Arch Ophthalmol. 122(6):827-837.

14. Budenz DL et al. (2005): Reproducibility of retinal nerve fiber thickness measurements using the Stratus OCT in 
normal and glaucomatous eyes. Invest Ophthalmol Vis Sci. 46(7):2440-2443.

15. Wollstein $\mathrm{G}$ et al. (2005): Comparison of three optical coherence tomography scanning areas for detection of glaucomatous damage. Am J Ophthalmol. 139(1):39-43.

16. Gyatso J et al. (2008): Retinal nerve fiber layer thickness in normal, Ocular hypertension and Glaucomatous Indian eyes. An optical coherence tomography study. J.Glaucoma 17(2):122-127.

17. Mahant A.J et al. (2011): Study of Retinal nerve fiber layer thickness with help of Optical Coherence Tomography in normal persons, Ocular hypertensive and Primary open angle glaucoma patients. Gujarat Medical Journal 66:16-21.

18. Jelena Kreso et al. (2008): glaucomatous parameters in normal, ocular hypertensive and glaucomatous eyes using optical coherence tomography. Acta Clin Croat 47(Suppl):39-42.

19. Bowd C et al. (2000): The retinal nerve fiber layer thickness in ocular hypertensive, normal, and glaucomatous eyes with optical coherence tomography. Arch Ophthalmol 118(1):22-26.

20. S. Subbiah et al. (2007): Comparative evaluation of optical coherence tomography in glaucomatous, ocular hypertensive and normal eyes. Indian journal of ophthalmology 55(4):243-247.

21. T Mansoori et al. (2010): Correlation between Peripapillary Retinal Nerve Fiber Layer Thickness and Optic Nerve Head Parameters Using Spectral Domain Optical Coherence Tomography Journal of Glaucoma: December 19(9):604-608.

22. Leung CK et al (2005): Comparison of macular and peripapillary measurements for the detection of glaucoma: an Optical Coherence Tomography Study. Ophthalmology. 112(3):391-400.

23. Sony P et al.(2004): Quantification of the retinal nerve fiber layer thickness in normal Indian eyes with optical coherence tomography. Ind J Ophthalmol. 52:303-309.

24. Ramakrishnan $\mathrm{R}$ et al. (2006): Retinal nerve fiber layer measurements in normal Indian population by optical coherence tomography. Ind J Ophthalmol. 54:11-15.

25. Schuman JS et al. (1995): Optical coherence tomography: a new tool for glaucoma diagnosis. Current Opin Ophthalmol. 6(2):89-95.

26. Budenz DL et al. (2005): Reproducibility of retinal nerve fiber thickness measurements using the Stratus OCT in normal and glaucomatous eyes. Invest Ophthalmol Vis Sci. 45:2440-2443.

27. Cook C1, Foster P. (2012): Epidemiology of glaucoma: what's new? Can J Ophthalmol. 2012 47(3):223-6. doi: 10.1016/j.jcjo.2012.02.003. 